\title{
SLENDERNESS EFFECTS IN EARTHQUAKE RESISTING FRAMES
}

\author{
A. L. Andrews*
}

\section{SYNOPSIS}

This study discusses lateral flexibility and displacement ductility controls which, if observed, will ensure that frame P-delta effects never become significant and so can be ignored. The controls are shown to be not too dissimilar from controls applied for other purposes. Thus they are tolerable. The study is linked to the New Zealand design rules. An appendix extends it to U.S. rules.

\section{INTRODUCTION}

Interaction between gravity loading and lateral deflection of frames gives rise to moments and forces additional to those determinable by traditional analysis. Of the various ways of compensating for analysis shortcomings, that of the American Concrete Institute is of most immediate interest because our current code(1) and its proposed replacement (yet unpublished) are both ACI oriented.

The ACI Code 318-7. $(2,3)$ commends to designers those analytical methods that take proper account of slenderness or P-delta effects. When it is impossible or impractical to analyse in this way, an augmentation procedure is to be used. Magnifiers which depend on the elastic properties of columns and of restraining members, on the distribution of moment in columns and on the durations of loadings are applied to moments.

This procedure is not appropriate for the design of earthquake resistant frames. In focussing on the role of compression members it tends to divert the designer's attention from the equal significance that slenderness effects have in beams. Concerned solely with elastic and creep strains, it fails to account for ductile hinging which is deliberately introduced into earthquake frame designs.

The provisions of ACI 318-71 which deal with unbraced frame moment augmentation (Sections 10.10 and 10.11) ought not be used in $\mathrm{N}$.w Zealand or anywhere else where seismicity is a concern and where the importance of ductile yielding as a force limiting phenomenon is understood and is used in design.

This note examines a prospect that lateral flexibility control, already a feature of enlightened earthquake design rules, can be adapted (if that is necessary) to ensure that slenderness, or "P-delta", effects will always be negligible. It turns out that controls imposed by the New Zealand Code $(4)$ are already sufficient in seismic Zone $A$ but that more restrictive limits than now apply are needed for Zones $B$ and $C$.

* Consulting Engineer, Wellington.

\section{A SIMPLE MODEL STRUCTURE}

The behaviour of a ductile frame can be reproduced on a simple model (Fig. la) consisting of a rigid load bearing strut hinged at its lower end and laterally supported at its upper end by an elastoplastic spring, elastic spring stiffness and elastic limit deflection $\delta_{e}$. In the absence of strut axial load this system responds to lateral load applied at spring level in a manner completely determined by the characteristic of the spring (Fig. lb) because the spring is the only loaded element. This behaviour matches the idealization customarily made of the elasticperfectly plastic response of a ductile frame to lateral load.

When the strut is required to support an axial load, $P$, concurrently with a horizontal load, $V$, which alone would cause the strut to assume an inclined position, the horizontal component of the strut load adds to the load in the spring. This is shown in Fig. Ic, a diagram of the strut equilibrium.

A study of this very simple system yeilds some useful information about the importance of $\mathrm{P}$-delta effects.

For equilibrium of the strut when the spring extension, $\delta$, is not more than the elastic Iimit extension, $\delta e^{\prime}$

$\delta \leqslant \delta e^{i} \quad \lambda \delta=V+\frac{P \delta}{h}$

and when $\delta$ exceeds the elastic limit extension

$\delta>\delta_{e} ; \quad \lambda \delta_{e}=V+\frac{p \delta}{h}$

There is a critical value, $\mathrm{P}_{\mathrm{Cr}}$, of the axial load for which the lateral stiffness of the system vanishes. At this critical load the spring extension can assume any arbitrary value equal to or less than the elastic limit extension. To find $\mathrm{P}_{\mathrm{Cr}}$, set $\mathrm{V}$ in equation (1a) at zero and obtain

$\mathrm{P}_{\mathrm{Cr}}=\lambda \mathrm{h}$

It is of incidental interest to observe how equation (2) compares with the equations of classical structural mechanics 
when used to predict critical loads for structures. Its worst performance is exemplified in the assessment of the critical load for a prismatic mast, Fig. (2a), for which the Euler load is well known to be

$P_{C r}=\frac{\pi^{2} E I}{4 h^{2}}=\frac{2 \cdot 47 E I}{h^{2}}$

The mast has a lateral stiffness (when no axial load is applied) of

$\lambda=\frac{3 E I}{h^{3}}$

whence, by equation (

$P_{\text {Cr }}=\frac{3 E I}{h^{2}}$

Comparison shows that equation (2) gives an error of overestimation of $22 \%$. This is a limiting error. For real frames, equation (2) usually gives better results as is shown by Fig. 2 (c), a comparison of results from precise analysis with results from equation (2).

Equations (1) can now be written in terms of $\mathrm{P}_{\mathrm{Cr}}$ thus:

For $\delta \leqslant \delta_{e} ; \quad V=\lambda \delta\left(1-\frac{P}{P_{C r}}\right)$
For $\delta>\delta_{e} ; \quad V=\lambda \delta_{e}\left(1-\frac{\delta P}{\delta_{e} P_{C r}}\right)$

The behaviour of the system determined by equations (3) is graphed in Fig. 3 (a). Two members of the family of curves, one of which is for zero axial load, are reproduced in Fig. 3 (b).

Consider now the work done by $\mathrm{V}$ acting through a displacement of $\mu \delta$ which corresponds to a displacement ductility factor $\mu$. When the axial load is zero, this work is represented by the area under the curve OAB of Fig. 3 (b) whence

$u_{0}=\frac{\lambda \delta e^{2}}{2}(2 \mu-1)$

The decrement, $\Delta \mathrm{U}$, caused by $\mathrm{P}$ is the area $O A B C D$. By noting that

$\begin{aligned} A D & =\frac{P}{P_{C r}} \lambda \delta_{e} \\ \cdot B C & =\frac{P}{P_{C r}} \mu \lambda \delta_{e}\end{aligned}$

the work decrement can be seen to be

$\left.\Delta U=\frac{1}{2} \frac{P}{P_{C r}} \lambda(\mu \delta)_{e}\right)^{2}$

This energy loss is the work done by $P$, or, in the case of a real structure, the loss of potential energy of the supported load, as can be seen by reference to Fig. 4, from which

$v=h-\left(h^{2}-\delta^{2}\right)^{\frac{1}{2}}=h-\left(h-\frac{\delta^{2}}{2 h}+\cdots \cdot\right)$

Therefore, to first order, $v=\frac{\delta^{2}}{2 h}$

Thus, the work done by $P$ is $\frac{P \delta^{2}}{2 h}$. By substituting for $h$ from equation (2) and putting $\delta$ at $\mu \delta_{e}$, there results:

Work done by $P=\frac{1}{2} \frac{P}{P_{C r}} \lambda\left(\mu \delta_{e}\right)^{2}=\Delta U$ by
equation (5).

It seems reasonable to limit this energy loss to a modest proportion, say 10\%, of the work done by $V$ in the absence of $P$, the latter being the assessment which would be made by a designer who had neglected "P-delta" effects in his analysis. The suggested criterion has it that ten times $\Delta U$ (eq. is not to exceed $U_{O}$ (eq. (4)). With the displacement ductility factor, $\mu$, at 4 , this reduces to

$P \leqslant 0.0438 \mathrm{P}_{\mathrm{Cr}}$

\section{APPLICATION TO FRAMES}

The critical load, $\mathrm{P}_{\mathrm{Cr}}$, for a frame is not easily found. It turns out to be better to calculate the energy decrement due to the descent of supported loads directly. By applying the result of equation (6) we obtain this expression for the vertical component of deflection, $v_{r}$, at the $r$ th floor in an n-storey building, axial shortening of the columns neglected:

$\nu_{r}=\frac{1}{2} \sum_{i=1}^{i=r} \frac{\left(\delta_{i}-\delta_{i-1}\right)^{2}}{h_{i}-h_{i-1}}$

in which $\delta_{i}$ is the total lateral deflection at floor $i$ and $h_{i}$ is the height of floor $i$ above some datum. Therefore the total loss of potential energy of supported loads in the building is

$\Delta U=\frac{1}{2} \sum_{r=1}^{r=n}\left(w_{r} \sum_{i=1}^{i=r} \frac{\left(\delta_{i}-\delta_{i-1}\right)^{2}}{h_{i}-h_{i-1}}\right)$.

in which $\mathrm{W}_{r}$ is the gravity load at floor $r$.

Suppose that the elastic flexibility of the frame is everywhere the greatest the design rules permit and thus is $k_{1}\left(h_{i}-h_{i-1}\right)$ where $K_{1}$ is a constant specified in the design rules. Suppose, further, that the displacement ductility demand, $\mu$, contemplated by the rules is everywhere simultaneously used. Then P-delta effects will be at their worst. If all storeys have the same height, $\mathrm{H}$, then equation ( 8 ) becomes:

$\Delta U=\frac{\mu^{2} \mathrm{~K}_{I}{ }^{2} \mathrm{H}}{2} \underset{\mathrm{I}=\mathrm{I}}{\mathrm{r}=\mathrm{n}} \mathrm{r} \mathrm{W}_{\mathrm{r}}$

Under the same conditions, but without considering the effects of axial load, the work done by an earthquake simulating force, $V_{r}$, acting at floor $r$ through a displacement $\mu \delta_{r}$ is given by

$U_{\text {or }}=\frac{1}{2} V_{r} \delta_{r}(2 \mu-1)=\frac{1}{2} v_{r} K_{I} r H(2 \mu-1)$

so the total work done by the system of forces $v_{r}$ is

$U_{0}=\frac{1}{2} K_{1} H(2 \mu-1) \underset{r=1}{r=n} r V_{r}$ 
With the same criterion for judging acceptability that was proposed earlier, we limit the decrement given by eq. (9) to 10\% of the work represented by eq. (10) to obtain

$10 \mu^{2} \mathrm{~K}_{I_{r=1}}^{r=n} r W_{r} \leqslant(2 \mu-1) \sum_{r=1}^{r=n} r V_{r}$

The system of earthquake simulating forces $V_{r}$ is related to the system of gravity forces $W_{r}$ by the design specification, according to which

$v_{r}=K_{2} \frac{r W_{r}}{n}$

in which $\mathrm{K}_{2}$ is a constant for the building, determined by the load distribution, the seismic zone and the period of free vibration in the fundamental mode. When this is substituted in (11), the control relationship reduces to

$10 \mu^{2} \frac{\mathrm{K}_{1} \mathrm{n} r=\mathrm{n}}{\mathrm{K}_{2}} \sum_{r=1} \mathrm{~V}_{r} \leqslant(2 \mu-1) \sum_{r=\mathrm{n}}^{r=\mathrm{n}} \mathrm{rV}_{r=1}$

Divide through by $\sum V_{r}$ and note that the quotient of $r=1$ summations on the right hand side now represents the height, in storeys, of the resultant of the forces $\mathrm{V}_{r}$. Call this height $\mathrm{n}_{\mathrm{g}}$ and obtain

$10 \mu^{2} \frac{\mathrm{K}_{1} \mathrm{n}}{\mathrm{K}_{2}} \leqslant(2 \mu-1) \mathrm{n}_{\mathrm{g}}$

If the building has, approximately, a uniform distribution of gravity load with height it will have an approximately triangular distribution of $V_{r}$ from zero at the ground to a maximum at the $n$th floor. For such a building $\mathrm{n}=1.5 \mathrm{n}_{\mathrm{g}}$ and $\mathrm{K}_{2}=2 \mathrm{C}_{\mathrm{d}}$ where $\mathrm{C}_{\mathrm{d}}$ is the code base shear coefficient, whence

$\frac{K_{1}}{C_{d}} \leqslant \frac{2(2 \mu-1)}{15 \mu^{2}}$

The drift Iimitation imposed by NZS 4203 is obtained from Table 10 and Clause 3.8.3, according to which

$\frac{\delta_{\mathrm{e}}}{\mathrm{H}}=\mathrm{K}_{1}=\frac{0.01}{v}=\frac{\mathrm{c}_{\mathrm{d}}}{200 \mathrm{CI}}$

where $C_{d}, C$, I have meanings assigned to them in the code. With this value for $K_{l}$, criterion (15) yields the following limit for $C$ :

$C \geqslant \frac{15 \mu^{2}}{400(2 \mu-1) I}$

This curious dependence of limit $C$ on I (importance factor) is a consequence of the arbitrary way the various factors which control design forces have been used in the code. Putting $\mu$ at 4 , which is said to be the displacement ductility factor that makes our pseudospectra appropriate, and I at its "usual" value of unity, we obtain

$C \geqslant 0.086$

When the coefficient $C$ is smaller than 0.086 control is not sufficiently tight to ensure that $\mathrm{P}$-delta energy decrement is less than 10\% of the zero $\mathrm{P}$ energy dissipating capacity. Because the capacity of ductile frames to survive intense earthquakes depends on their ability to dissipate energy, it is necessary to observe some such limitation as has been suggested in these notes. In practical terms there are no problems in Seismic zone $A$, but there may be problems in Zone $B$ for structures with fundamental vibration periods greater than $0.9 \mathrm{sec}$. and in Zone $C$ for structures with periods exceeding $0.65 \mathrm{sec}$. The New Zealand pseudospectra are known to be very conservative for long period buildings.

\section{EFFECT OF P-DELTA ELASTIC SOFTENING}

There is a secondary consideration neglected in the foregoing development, to be found in p-delta softening in the elastic range. This softening increases the fundamental vibration period and thus reduces the base shear coefficient appropriate for the structure. The base shear reduction has not been accounted for. Refer to equations (3) and to the diagrams of Fig. 2 from which

$\frac{\text { Elastic lateral stiffness }}{\text { Elastic lateral stiffness at } \mathrm{P}=0}=1-\frac{\mathrm{P}}{\mathrm{P}_{\mathrm{Cr}}}$
Whence $\mathrm{T}=\mathrm{T}_{\mathrm{O}} \sqrt{\frac{1}{1-\frac{\mathrm{P}}{\mathrm{P}}}}=\mathrm{T}_{\mathrm{Cr}}\left(1+\frac{\mathrm{P}}{2 \mathrm{P}_{\mathrm{Cr}}}+\ldots.\right)$

so $\mathrm{T}=\mathrm{T}_{0}\left(1+\frac{\mathrm{P}}{2 \mathrm{P}_{\mathrm{Cr}}}\right)$ to first order. Here

$T$ is the fundamendal vibration period and $T_{0}$ is the assessment of it when p-delta effects are ignored.

But $\frac{\mathrm{P}}{\mathrm{P}_{\mathrm{Cr}}}=\frac{\mathrm{P}}{\lambda \mathrm{h}}=\frac{\mathrm{P} \delta}{\mathrm{Vh}}=\frac{\delta_{\mathrm{e}}}{\mathrm{C}_{\text {do }} \mathrm{h}}$ in which $\mathrm{C}_{\text {do }}$ is the value of $C_{d}$ appropriate for the system with a stiffness which does not account for p-delta, so

$\mathrm{T}=\mathrm{T}_{0}\left(1+\frac{\delta_{\mathrm{e}}}{2 \mathrm{C}_{d_{0}} \mathrm{~h}}\right)$ to first order.

As before when the flexibility is maximised, we have a limit of $\frac{\nu \delta_{e}}{h}$ at .01 and $v$ is $\frac{2 C_{0} I}{C_{d o}}$

according to the code, zero subscripts indicating zero axial load assessments as before, so

$\mathrm{T}=\mathrm{T}_{\mathrm{O}}\left(1+\frac{1}{400 \mathrm{C}_{\mathrm{O}} \mathrm{I}}\right)$

For Zone $C$, firm ground, the sloping portion of the pseudospectrum, which is the only part to interest us in view of the conservatism of high period values, is represented by

$T=15(0.13-C)$

Now, putting I at its usual value of unity, we obtain

$c=c_{0}+\frac{C_{0}-0.13}{400 c_{0}}$

Equation (18) gires the base shear coefficient in terms of the coefficient which would be obtained from an analysis which neglected 
P-delta. From it we see that the percent error of overestimation when P-delta effects are not included in the analysis for the most flexible of permitted frames is

Error $\frac{0.13-\mathrm{C}_{0}}{4 \mathrm{C}_{0}{ }^{2}}, 0.45 \mathrm{sec} . \leqslant \mathrm{T}_{0}<1.2 \mathrm{sec}$.

Bearing in mind that neglect of p-delta. effects may cause about a $20 \%$ overestimation of the work done by earthquake simulating forces in a Zone $C$ structure with a fundamental period of about $1.2 \mathrm{sec}$., this error is seen to be insignificant and does not compensate, as the following table shows:

$$
C_{0} \text { from code }
$$

(Seismic Zone C)

$0.10 \quad(T=0.449 \mathrm{sec})$
$0.10 \quad(T=0.45 \mathrm{sec})$
0.09
0.08
0.07
0.06
0.05

\section{RECOMMENDATION}

Deflection control provides an efficient, acceptable and certain means of limiting P-delta effects to tolerable levels. Current design controls for Zone A suffice to do this; but more stringent control is needed for zones $B$ and $C$. For these zones lateral stiffness should match that required of buildings in Seismic zone $A$. This would be achieved by making the absolute deflection limit specified in Clause 3.8 .3 seismic zone dependent, leaving its current value of 0.01 for Zone $A$ and introducing new limits of .008 and .006 for seismic zones $B$ and $C$ respectively.

\section{REFERENCES}

1. Standards Association of New Zealand, "Code of Practice for Reinforced Concrete - Design", NZS 3101P:1970.

2. American Concrete Institute, "Building Code Requirements for Reinforced Concrete (ACI 318 - 71)".

3. American Concrete Institute, "Commentary on Building Code Requirements for Reinforced Concrete (ACI 318 - 71)"

4. Standards Association of New Zealand, "Code of Practice for General Structural Design and Design Loadings for Buildings", NZS 4203 : 1976

5. Seismology Committee, Structural Engineers Association of California, "Recommended Lateral Force Requirements and commentary", 1974

\section{APPENDIX}

\section{APPLICATION IN AREAS WHERE SEAOC RULES ${ }^{(5)}$}

\section{APPLY}

To and including the establishment of a limit base shear (equation (17)) the development is almost the same as it is for New Zealand. There are some notation differences, but much the same sort of deflection control applies, and the same displacement ductility is implied. The SEAOC lateral load intensity is differently specified. When this difference is considered, it turns out that there may be problems in the most intensely seismic of SEAOC zones for structures having fundamental vibration periods which exceed $0.74 \mathrm{sec}$. For zones of less intense seismicity, P-delta effects will be significant in shorter period buildings. ( $T_{S}$ has been taken to be 1.5 sec.).

In considering whether P-delta softening may compensate by an appropriate lowering of the earthquake simulating lateral load, we have, for a typical building in $\mathrm{z}=1$ Zone, $\mathrm{T}_{\mathrm{S}}$ at $1.5 \mathrm{sec}$. as before,

$C S=\frac{0.1}{\sqrt{T}}$

which, when used to eliminate $T$ and $T_{0}$ from the expression previously developed for P-delta softening gives

$\frac{1}{\mathrm{C}^{2} \mathrm{~s}^{2}}=\frac{1}{\mathrm{C}_{0}^{2} \mathrm{~s}_{0}^{2}}\left(1+\frac{1}{400 \mathrm{C}_{0} \mathrm{~s}_{0}}\right)$

whence

$C S=C_{0} S_{0}\left(1+\frac{1}{400 C_{0} S_{0}}\right)^{-\frac{1}{2}}$

Form the binominal expansion of the bracketed term on the right hand side and retain as significant only the first two terms to get

$C S=C_{0} S_{0}-\frac{1}{800}$

from which it is clear that the decrement in earthquake simulating load which can be justified by P-delta softening is negligible.

If SEAOC limits are to serve to control P-delta they need some tightening. Nevertheless, it would be preferable to impose stricter controls than to oblige designers to deal with complex interaction effects.

Paper received 2 september, 1977. 


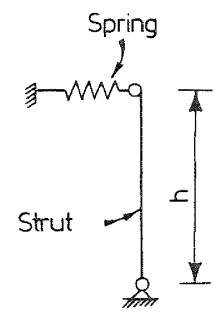

Strut and Spring

(A)

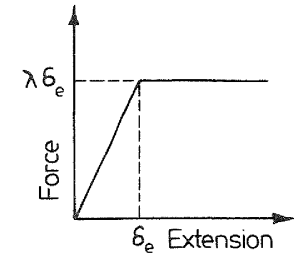

Spring Character

(B)

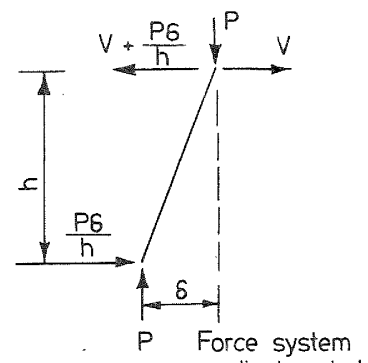

on displaced strut

FIGURE 1

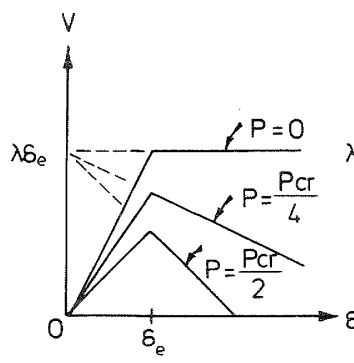

(A)

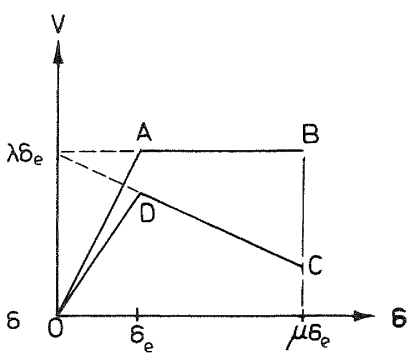

(B)

FIGURE 3

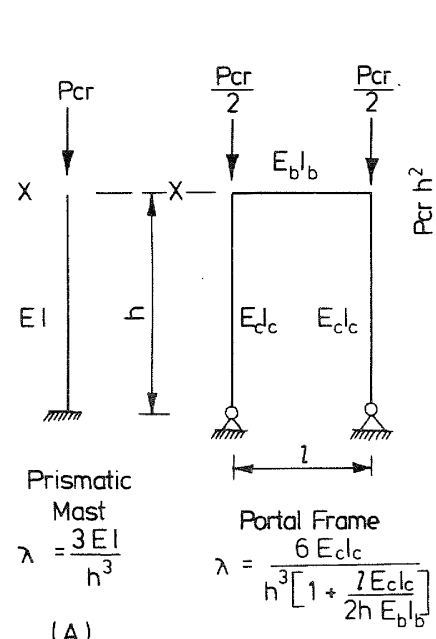

(B)

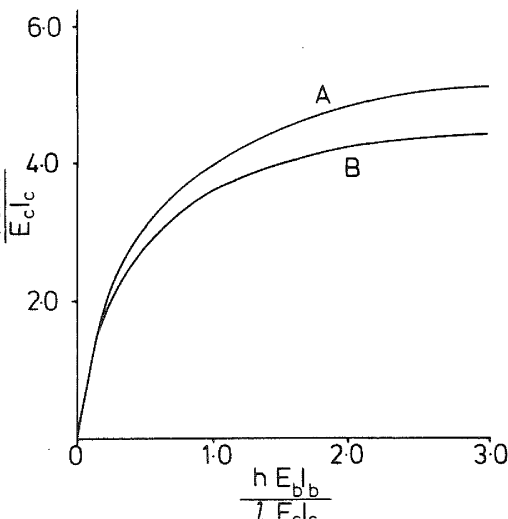

Instability Loads for Portal Frame

A - Estimate by Eq. (2)

$B-B y$ theory of elastic stability

(C)

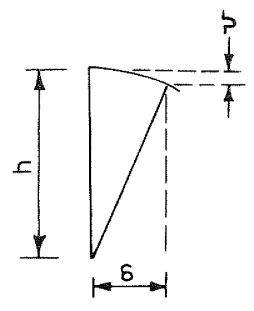

FIGURE 4

In $(A)$ and $(B) \lambda$ is the lateral force at level $X$ needed to cause a unit lateral displacement there, axial load effects neglected

FIGURE 2 